\title{
Permutations in Coinductive Graph Representation
}

\author{
Celia Picard and Ralph Matthes* \\ Institut de Recherche en Informatique de Toulouse (IRIT), \\ University of Toulouse and C.N.R.S., France
}

\begin{abstract}
In the proof assistant Coq, one can model certain classes of graphs by coinductive types. The coinductive aspects account for infinite navigability already in finite but cyclic graphs, as in rational trees. Coq's static checks exclude simple-minded definitions with lists of successors of a node. In previous work, we have shown how to mimic lists by a type of functions and built a Coq theory for such graphs. Naturally, these coinductive structures have to be compared by a bisimulation relation, and we defined it in a generic way.

However, there are many cases in which we would not like to distinguish between graphs that are constructed differently and that are thus not bisimilar, in particular if only the order of elements in the lists of successors is not the same. We offer a wider bisimulation relation that allows permutations. Technical problems arise with their specification since (1) elements have to be compared by a not necessarily decidable relation and (2) coinductive types are mixed with inductive ones. Still, a formal development has been carried out in Coq, by using its built-in language for proof automation.

Another extension of the original bisimulation relation based on cycle analysis provides indifference concerning the root node of the term graphs.
\end{abstract}

\section{Introduction}

In 16, we have developed a complete solution to overcome guardedness issues regarding programming and subsequent verification with embedded lists in a coinductive type in the proof assistant Coq [19], with the aim of graph representation (we represent single-rooted, connected graphs). In the present article, we present a more versatile bisimulation relation over those graphs than the one studied in [16] that naturally followed from the obtained coinductive representation of graphs.

First of all, we give a brief summary of the main notions introduced in [16] that will be needed for the rest of the article. Although all of this reports on formalization work that has been carried out in Coq, we refrained from displaying Coq syntax (the Gallina language) or even of its underlying type theory the calculus of inductive and coinductive constructions (CIC). We try to adopt

\footnotetext{
* This work has been funded by the project CLIMT of the French Agence Nationale de la Recherche (ANR-11-BS02-016).
} 
standard mathematical or standard type-theoretical notation as much as possible. In case of doubt what the exact correspondence is with the $\mathrm{CIC} / \mathrm{Coq}$, we invite the reader to refer to our full proofs [17.

\subsection{Summary of the Notions Introduced in [16]}

The goal we were aiming at was coinductive graph representation. We explained that we needed a structure that would mimic lists without being inductive (this was necessary because of Coq's guardedness condition (see [4] and [1]) which is based on a productivity criterion (see [7])). Therefore, we decided to use functions (this idea has also been mentioned by Chlipala in [5]). The domain of definition of those functions is a set of $n$ elements, $n$ being the length of the list we want to mimic. It is defined inductively through the two following constructors:

\section{Definition 1 (Fin, Viewed Inductively)}

$$
\frac{n: \mathbb{N}}{\text { first } n: \operatorname{Fin}(n+1)}
$$

$$
\frac{n: \mathbb{N} \quad i: \text { Fin } n}{\text { succ } i: \text { Fin }(n+1)}
$$

This definition has also been used by Altenkirch in 1 and by McBride and McKinna in 12. By construction, Fin $n$ is a type with precisely $n$ elements. It actually corresponds to the container view of lists [18].

Remark 1 (Notations). In the rest of the paper we will use the following notations:

- T, $U$ for types and $t$ (resp. $u$ ) for elements of type $T$ (resp. $U$ ),

$-n, m$ and $k$ for elements of $\mathbb{N}$,

$-l$ and $q$ for lists and elements of ilist ("indexed lists"), to be defined below,

- $f$ for functions,

- $g$ for elements of Graph, to be defined below,

- $R$ for binary (endo-)relations: a relation on $T$ has type $T \rightarrow T \rightarrow$ Prop, with $\rightarrow$ right-associative (as always in this paper) and Prop the Coq universe of propositions (in standard mathematics, one would just have the two truth values in Prop, and the informal semantics of relations is the standard one, but the application notation $R t_{1} t_{2}$ is used instead of $R\left(t_{1}, t_{2}\right)$ in standard mathematics),

- $i$ for elements of Fin $n$,

- if a relation $S$ depends on a relation argument $R$, we say that $R$ is the base relation of $S_{R}$, and that $S$ preserves equivalence if, for every equivalence relation $R, S_{R}$ is an equivalence relation (likewise with reflexivity, symmetry and transitivity alone).

The type of functions that mimics lists of length $n$ is defined as follows:

Definition 2. ilistn $T n:=$ Fin $n \rightarrow T$

However, this is not yet satisfactory as ilistn still has the length parameter $n$ while lists do not - it is inherent to them. Therefore, we define the following structure consisting of the length and a function in the corresponding ilistn: 
Definition 3. ilist $T:=\Sigma n: \mathbb{N}$. ilistn $T n$

$\Sigma$ denotes a strong sum type (of pairs where the type of the second component depends on the first component). Consequently, the elements of ilist $T$ are written as pairs $\langle n, \ln \rangle$ with $l n$ of type ilistn $T n$. We call the associated projection functions $l g$ and $f c t$, i. e., $l g\langle n, l n\rangle=n$ and $f c t\langle n, l n\rangle=l n$. One can show that ilist is in pointwise bijection with lists that are preloaded from the standard library in Coq (to do so, we use two conversion functions ilist2list and list2ilist and show that their compositions are pointwise equal to the identity - since Coq lacks functional extensionality, this does not imply equality with the identity).

Remark 2. In the container view, $n$ corresponds to the shape of the container, and Fin $n$ to the type of positions (an element of Fin $n$ is a position).

We need to lift relations on $T$ to relations on ilist $T$ expressing that the base relation holds element-wise. In our intended use, the type $T$ will be the coinductive type for graphs on which Leibniz equality - the propositional equality = of Coq - cannot be used. We define the following generic operation ilist_rel:

Definition 4 (ilist_rel)

$$
\begin{aligned}
\forall l_{1} l_{2}: \text { ilist T, ilist_rel } l_{R} l_{1} l_{2} \Leftrightarrow & \exists h: \lg l_{1}=\lg l_{2}, \\
& \forall i: \text { Fin }\left(\lg l_{1}\right), R\left(\text { fct } l_{1} i\right)\left(\text { fct } l_{2} i_{h}^{\prime}\right)
\end{aligned}
$$

where $i_{h}^{\prime}$ is the result of converting $i$ to type Fin $\left(\lg l_{2}\right)$ using hypothesis $h$.

This conversion is needed since the theoretical basis of Coq - the CIC - is an intensional type theory, where equal types cannot enter the type-checking process more deeply than equal terms, i. e., if a term $t$ has type $A$ and one can prove $A=B$, the term $t$ does not have type $B$, but there is a construction taking $t$ and yielding an inhabitant of $B$. For more details about the pattern matching feature that allows us to make this type conversion in Coq, see 19, Chap. 1.2.13 and 4.5.4]. In this paper, we do not expose the technical issues with type conversion that have to be mastered in the reasoning involving ilist_rel.

We define the type of graphs of interest in our paper through the constructor:

\section{Definition 5 (Graph, Viewed Coinductively). $\frac{t: T \quad l: i l i s t(G r a p h T)}{m k_{-} G r a p h t l: G r a p h T}$}

Coinductive types in Coq are written with their constructors, just as for inductive types although categorical duality would suggest to define coinductive types by their destructors (projection functions). The constructor-based format is usually preferred for programming purposes. In order to distinguish the constructorbased view of coinductive definitions from inductive definitions, we put a double line in the inference rules. This notation is also used in [14]. Call label and sons the two projection functions on Graph, i. e., label $\left(m k_{-} G r a p h t l\right)=t$ and sons $\left(m k_{-} G\right.$ raph $\left.t l\right)=l$. They correspond to the unfolding of the greatest fixed point and are more basic than the constructors in coalgebraic approaches.

Had we used lists instead of $i$ ilst, we would have had an embedded inductive type in a coinductive type, with all the problems of guardedness as described in [16]. 
However, another approach to solve the same kind of problem in Coq has been proposed by Dams in [8], where the embedded inductive type is even extended to a coinductive one. This solution seems to be quite heavy to manipulate as there are a lot of proofs to carry out. Niqui in [15] and Bertot and Komendantskaya in 4. propose solutions also for a coinductive embedded type (and not an inductive one). The latter solution is quite close to ours, but on streams instead of lists, and hence without the need to restrict to a finite domain. For the finite domain, we chose Fin $n$, and thus "objects" $n$ enter types and we are forced to use techniques of dependently-typed programming. Moreover, we have to quantify existentially over $n$, and this constructively, as is done with the $\Sigma$-type.

The same kind of issues also appears in other proof assistants, e. g., in Agdd 1 , even though it has recently seen quite some progress in its termination checker. In 9] Danielsson describes an experimental solution to solve them (see also the extended case study with Altenkirch in [10]). This extension by datatypes that may have both inductive and coinductive constructors is still experimental. In Coq, this is just not available.

We lift relations $R$ on $T$ to relations $G e q_{R}$ on Graph $T$ in a generic manner:

\section{Definition 6 ( $G e q$, Viewed Coinductively)}

$g_{1} g_{2}:$ Graph $T \quad R\left(\right.$ label $\left.g_{1}\right)\left(\right.$ label $\left.g_{2}\right) \quad$ ilist_rel $_{G e q_{R}}\left(\right.$ sons $\left.g_{1}\right)\left(\right.$ sons $\left.g_{2}\right)$

$\mathrm{Geq}_{R} g_{1} g_{2}$

It becomes apparent that ilist_rel $_{R}$ had to be defined for arbitrary relations, not only Leibniz equality. Preservation of equivalence by ilist_rel cannot suffice to prove that $G e q$ preserves equivalence, but still, preservation holds [16].

Remark 3. The two graphs on the right are equivalent through Geq. If one wanted to differentiate between these two structures, one could use the type $\{A, B\} \times \mathbb{N}$ instead of $\mathbb{N}$ and thus give an identity to the nodes.
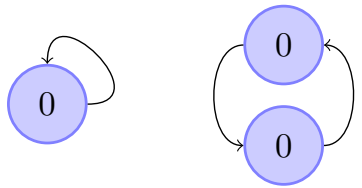

\subsection{Need for a More Liberal Relation on Graph - Content Overview}

With Geq, we are forced to give an order to the nodes, horizontally and vertically, while in a classical set-theoretic graph representation, there is no such order. We aim at obtaining the more liberal equivalence of the classical representation on our constructive representation, e. g., we want the graphs of Fig. 1 or of Fig. 2] to be considered equivalent. However, with respect to Geq, they are not (here and in all further examples, we implicitly use Leibniz equality = as the base relation). Indeed, their nodes are not in the same order. In the case of Fig. 1, the children of the root are inverted in the two graphs (different "horizontal" order). In Fig. 2 the second graph has been turned by $180^{\circ}$ (different "vertical" order). The new equivalence relation should solve the two cases illustrated by Fig. 1 and Fig. 2 , The first one corresponds to a permutation between the children of an element

${ }^{1}$ http://wiki.portal.chalmers.se/agda/pmwiki.php 
of Graph. Thus, this corresponds also to a new equivalence relation on ilist (that indicates that two elements of ilist are permutations of each other). The second one corresponds to a change in the point of view of the observation of the graph.
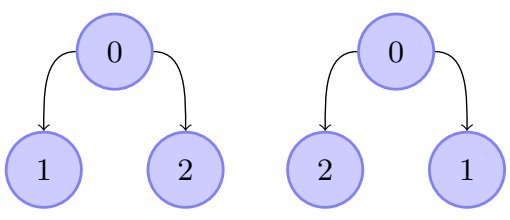

Fig. 1. Different order in children
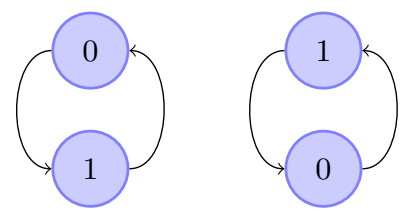

Fig. 2. Different roots

This paper presents the development of these new relations. In Sect.2 we present and compare various ways to represent permutations on ilist. Sect. 3 uses our favourite one to define relation GPerm on Graph to capture permutations of children (see Fig. 1), with an extended discussion about how to interpret the coinductively defined relation. Sect.4shows a characterization of GPerm by a sequence of relations corresponding to increasingly deep observations of the graphs involved. Finally, in Sect.5 we present a relation on Graph that solves the two issues mentioned above (corresponding to the features of Fig. 1 and Fig. 2).

All the ideas presented in the body of this paper are original with respect to [16. All of the contents of this article has been formally modeled and proved in Coq (version 8.3). The development is available in [17.

\section{Capturing Permutations on ilist}

A first and standard solution would be to check that the elements of ilist we are comparing contain the same number of occurrences of each element. However, counting occurrences needs decidability of $R$, i. e., $\forall t_{1} t_{2},\left(R t_{1} t_{2}\right) \vee \neg\left(R t_{1} t_{2}\right)$, with a strongly constructive form $\vee$ of disjunction that comes with evidence for the case that has been proven (in Coq, this is sumbool, which belongs to the universe Set of computationally relevant types).

The relation that ensures the same numbers of occurrences of all elements cannot replace ilist_rel in the definition of Geq as its index relation is the one that we would be defining, and for which decidability cannot be already known. Anyway, a posteriori, decidability for Geq cannot be expected.

We could turn to an inductive characterization of whether an element of ilist has a certain number of occurrences. Instead, we preferred to give a direct inductive characterization of when permutations exist. In the next section we will give two equivalent such characterizations, and in Sect.2.2 we will enrich with "witnesses" one of these. In Sect.2.3, we give a declarative notion that does not depend on induction. In Sect.2.4, we give a formal comparison of these notions, while we finish with a more informal discussion in Sect.2.5. 


\subsection{Inductive Definitions of Permutations on ilist}

We now explore notions of permutations that do not depend on decidability of the base relation. Ideally, we want to remain in the realm of logical/declarative approaches. Instead of saying what a permutation is, we will specify when it exists. We do this constructively - by an inductive generation process. Read backwards, we basically remove equivalent elements one by one.

To do so, we need a function that removes an element from an ilist. The idea is that it only keeps the elements "before" and "after" the one we want to remove. We call this function remEl but we do not give a formal definition here because it is rather technical and we want to keep the article free from difficulties of dependently-typed programming. It has type remEl $(l:$ ilist $T):$ Fin $(l g l) \rightarrow$ ilist $T$. It is characterized by the following assertions:

$$
\begin{aligned}
& \lg (\text { remEl }\langle n+1, \ln \rangle i)=n \\
& i^{\prime}<_{\text {Fin }} i \Rightarrow \operatorname{fct}(\operatorname{remEl}\langle n+1, \ln \rangle i) i^{\prime}=\ln \left(\text { weakFin } i^{\prime}\right) \\
& i \leq_{\text {Fin }} i^{\prime} \Rightarrow \operatorname{fct}(\operatorname{remEl}\langle n+1, \ln \rangle i) i^{\prime}=\ln \left(\operatorname{succ} i^{\prime}\right)
\end{aligned}
$$

where weakFin $: \forall n$, Fin $n \rightarrow$ Fin $(n+1)$ is recursively defined by: weakFin $($ first $n):=\operatorname{first}(n+1)$ and weakFin $\left(\operatorname{succ} i^{\prime}\right):=\operatorname{succ}\left(\right.$ weakFin $\left.i^{\prime}\right)$ This function only increases the type indices in its argument. Logically speaking, this is a kind of weakening. Please, note that 2 and 3 only type well modulo 1

Remark 4 (Order relation on Fin). We informally use $<_{\text {Fin }}, \leq_{\text {Fin }}$ and $=_{\text {Fin }}$ to represent the order relation on Fin. Basically, we order the elements of Fin by the number of succ in their definition, disregarding the type indices, e.g., first $2<_{\text {Fin }} \operatorname{succ}($ first 1$)=_{\text {Fin }} \operatorname{succ}($ first 2$)$. Thus, elements of different types are put into relation. One can show $\forall i$,weakFin $i={ }_{F i n} i$.

The permutation relation can be defined using any one of the two following definitions (we will prove that they are equivalent):

\section{Definition 7 (iperm, Viewed Inductively)}

$$
\forall l_{1} l_{2}, \text { iperm }_{R} l_{1} l_{2} \Leftrightarrow\left\{\begin{aligned}
l g l_{1}=l g l_{2}=0 \quad \text { or } \\
\exists i_{1} i_{2}, R\left(\text { fct } l_{1} i_{1}\right)\left(f c t l_{2} i_{2}\right) \\
\wedge \operatorname{iperm}_{R}\left(\text { remEl } l_{1} i_{1}\right)\left(\text { remEl } l_{2} i_{2}\right)
\end{aligned}\right.
$$

\section{Definition 8 (iperm' , Viewed Inductively)}

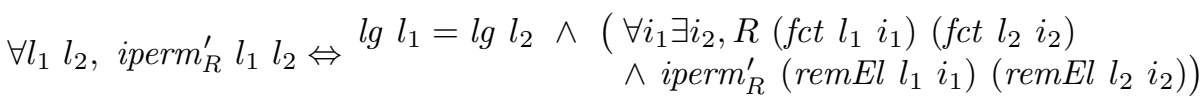

Note that, iperm gives us one pair of equivalent elements that we can remove at each "level", while iperm' says that at each level all elements of $l_{1}$ have an equivalent one in $l_{2}$ (and this recursively). Roughly, iperm can be seen as a particular case of iperm ${ }^{\prime}$ since, if $l g l_{1}>0$, there is the canonical choice of $i_{1}$ to be the first element of its domain. 
Before proving the equivalence of these definitions, we observe that the following assertion: $\forall l_{1} l_{2}$, iperm ${ }_{R} l_{1} l_{2} \Rightarrow l g l_{1}=l g l_{2}$ holds, to be proven by induction.

Theorem 1. $\forall l_{1} l_{2}$, iperm $_{R} l_{1} l_{2} \Leftrightarrow$ iperm $_{R}^{\prime} l_{1} l_{2}$

Proof

$\left[\right.$ iperm $^{\prime} \Rightarrow$ iperm $]$ This was already discussed. Formally, it is done by induction on iperm'.

[iperm $\Rightarrow$ iperm $\left.{ }^{\prime}\right]$ To prove this, we reason by induction on $l g l_{1}$.

- If $l g l_{1}=0$ then $l g l_{2}=0$ (using the above observation), hence iperm $^{\prime}$ is trivially true since there is no $i_{1}$ : Fin 0 .

- If $l_{1}$ and $l_{2}$ are not empty, the proof is much harder. As we already know that $\lg l_{1}=\lg l_{2}$, what we have to prove is:

$$
\begin{aligned}
\text { iperm }_{R} l_{1} l_{2} \Rightarrow\left(\forall i_{1} \exists i_{2},\right. & R\left(f c t l_{1} i_{1}\right)\left(f c t l_{2} i_{2}\right) \wedge \\
& \text { iperm } \left.{ }_{R}^{\prime}\left(\text { remEl } l_{1} i_{1}\right)\left(\text { remEl } l_{2} i_{2}\right)\right)
\end{aligned}
$$

Its proof is quite subtle because it requires a precise case analysis on the index $i_{1}$, which is complicated in this dependently-typed setting. A crucial role is played by interchange lemmas for two subsequent applications of remEl. For its proof, one has to think of a complete unfolding of iperm until the base case is reached. Then, one may permute the order of the obtained pairs in the relation $R$, just as in the classical mathematical theory of permutations.

The usefulness of having these two equivalent definitions is that they are quite different in structure. Therefore, some properties are easier to show on one or the other. In particular, this is the case for the proof that iperm and iperm ${ }^{\prime}$ preserve equivalence: symmetry is easier with Definition 7 while transitivity is easier with Definition 8 (for $i_{1}$ in $l_{1}$, one gets $i_{2}$ in $l_{2}$ and then $i_{3}$ in $l_{3}$, while Definition 7 yields two possibly different indices in $l_{2}$ ). These proofs are not detailed here.

We have also proved that iperm (resp. iperm ${ }^{\prime}$ ) preserves decidability (see the definition of decidability in the introduction of Sect.22) :

Lemma 1. If $R$ is decidable, then iperm ${ }_{R}$ (resp. iperm ${ }_{R}^{\prime}$ ) is decidable.

Proof. One possibility is a proof by induction using Definition 8 (and Theorem[1).

\subsection{A Richer Notion of Inductive Permutations}

However, sometimes, knowing that a permutation exists will not be enough, for example when considering intersection of base relations, as in Lemma 3 below. Definitions 7 and 8 do not allow us to manipulate permutations directly since only their existence is specified. Thus, we have to enrich our definition with a notion of skeleton that witnesses the existence of a permutation. The skeleton records the choices for the existentially quantified variables according to Definition 7 Hence, each skeleton consists of a tuple of pairs of elements of Fin. In each pair, the first element of Fin corresponds to an index in the first ilist, and the second one to the corresponding element in the second ilist. These skeletons form types skel_type $n$, defined as $\mathbb{N}$-indexed family of types: 


\section{Definition 9 (skel_type, Defined Recursively)}

$$
\begin{aligned}
& \text { skel_type } 0:=\text { unit } \\
& \text { skel_type }(n+1):=(F i n(n+1) \times \text { Fin }(n+1)) \times \text { skel_type } n
\end{aligned}
$$

Here, unit is a one-element type (one might take unit $:=$ Fin 1 , but unit has nothing to do with indices in ilist).

Now, we can define a new notion of permutation (let's call it iperm_skel) using this notion of skeletons. Definition 8 would certainly not fit well with it, but Definition 7 does. To be able to use skel_type, we need one natural number as index for both components of the pair, in other words, we need to know that $\lg \left(\right.$ sons $\left.g_{1}\right)=\lg$ (sons $\left.g_{2}\right)$. Therefore, we have chosen to add this as the hypothesis $H_{l g}$ for iperm_skel. However, this is reasonable as we know that it is a direct consequence of iperm (as observed right after the definition of iperm ${ }^{\prime}$ ). We define iperm_skel in lockstep with Definition [7, but with the extra data:

\section{Definition 10 (iperm_skel, Viewed Inductively)}

$$
\begin{aligned}
& \forall l_{1} l_{2} H_{l g t i} s, \text { iperm_skel } l_{R} l_{1} l_{2} H_{l g t i} s \Leftrightarrow \\
& \left\{\begin{array}{l}
l g l_{1}=0 \text { or } \\
\exists i_{1} i_{2} s^{\prime}, R\left(\text { fct } l_{1} i_{1}\right)\left(\text { fct } l_{2} i_{2}\right) \wedge ~ “ s=\left(\left(i_{1}, i_{2}\right), s^{\prime}\right) " \wedge \\
\text { iperm_skel }_{R}\left(\text { remEl } l_{1} i_{1}\right)\left(\text { remEl } l_{2} i_{2}\right) H_{l g t i}^{\prime} s^{\prime}
\end{array}\right.
\end{aligned}
$$

where $H_{l g}$ is the aforementioned variable assuming $l g l_{1}=\lg l_{2}$ and is used to build a proof $H_{\text {lgti }}^{\prime}$ of lgti $\left(\right.$ remEl $\left.l_{1} i_{1}\right)=\operatorname{lgti}\left(\right.$ remEl $\left.l_{2} i_{2}\right)$, s is of type skel_type $\left(l g l_{1}\right)$ and " $s=\left(\left(i_{1}, i_{2}\right), s^{\prime}\right)$ " is only correct up to several type conversions that are not detailed here. Notice that $i_{1}$ is of type Fin $\left(\lg l_{1}\right), i_{2}$ is of type Fin $\left(\lg l_{2}\right)$ and $s^{\prime}$ is of type skel_type $\left(\lg \left(\right.\right.$ remEl $\left.\left.l_{1} i_{1}\right)\right)$.

We can show that iperm and iperm_skel are equivalent (proof not given here):

Lemma 2 (iperm $\Leftrightarrow$ iperm_skel)

$$
\forall l_{1} l_{2} H_{l g}, \text { iperm }_{R} l_{1} l_{2} \Leftrightarrow \exists s, \text { iperm_skel }_{R} l_{1} l_{2} H_{l g} s
$$

We can also show that iperm_skel is monotone in its relation argument (this was also true for iperm). Finally, we also obtain a lemma about relation intersection for iperm_skel given a specific skeleton:

Lemma 3 (Intersections in the Relation Argument of iperm_skel). For fixed $l_{1}, l_{2}, H_{l g}$ and skeleton $s$, iperm_skel $l_{R} l_{1} l_{2} H_{l g t i} s$ commutes with arbitrary intersections of non-empty sets of relations $R$. In particular, if for all $n$ iperm_skel $_{R_{n}} l_{1} l_{2} H_{l g t i} s$, then iperm_skel $\cap_{n} R_{n} l_{1} l_{2} H_{l g t i} s$.

Note that, without the skeleton - for iperm - this cannot be expected. This lemma will be necessary for the proof of Lemma 10 (see Sect.4.2). 


\subsection{Permutations on ilist with Bijective Functions}

The previous solution is constructive and does not require decidability but remains inductive. Hence, when we mix it with a coinductive definition on Graph (when defining an equivalence relation on Graph, for instance), we will have the same kind of problems as the ones we had when mixing lists with Graph (even though, as we will see in Sect.3. since we are in the universe of propositions - not of sets, we have more ways to overcome the guardedness restrictions). Thus, we present here a last solution that does not require decidability and that is not inductive. It is based on an idea similar to the one we used for ilist as we use functions. This solution says that two elements of ilist are permutations of each other if there is a bijective function from the indices of the first one to the indices of the second one and when each pair of indices in relation points to equivalent elements.

First of all, we decide to express that a function is bijective by the following definition (where one has to give explicitly the inverse of the function) :

Definition 11 (bij)

$$
\forall(f: T \rightarrow U)(g: U \rightarrow T), \text { bij } f g \Leftrightarrow(\forall t, g(f t)=t) \wedge(\forall u, f(g u)=u)
$$

Now we can define the permutation relation using bij:

\section{Definition 12 (ipermb)}

$$
\forall l_{1} l_{2}, \text { ipermb }_{R} l_{1} l_{2} \Leftrightarrow \exists f g, \text { bij } f g \wedge\left(\forall i, R\left(f c t l_{1} i\right)\left(f c t l_{2}(f i)\right)\right)
$$

Remark 5. Note that ipermb has exactly the same logical structure as ilist_rel. Actually, ilist_rel can be seen as a special case of ipermb.

One easily shows that ipermb preserves equivalence, that it is monotone in its base relation and the following minimal requirement for a definition of permutation:

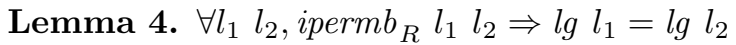

\subsection{Equivalence Proofs between the Definitions}

In order to validate Definitions 7 and 12, we show that they are equivalent and that they are equivalent to a permutation relation on lists given by Contejean [6] (and thanks to Theorem 1 this will also validate Definition 8).

Equivalence between iperm and ipermb. These definitions have the same coverage, in particular, they do not require decidability of $R$, and they achieve the same objective. We will show here that they are equivalent.

Theorem 2. $\forall l_{1} l_{2}$, iperm $_{R} l_{1} l_{2} \Leftrightarrow$ ipermb $_{R} l_{1} l_{2}$

Proof

[Direction $\Rightarrow$ ] Actually here, what we really do is to prove $i p e r m b_{R} l_{1} l_{2}$ from iperm_skel $l_{R} l_{2}$ (thanks to Lemma 2). We obtain the two functions needed for ipermb from the skeleton provided by iperm_skel. Then we finish the proof (for the $\forall i, R\left(f c t l_{1} i\right)\left(f c t l_{2}(f i)\right)$ part) by induction on $l g l_{1}$. 
[Direction $\Leftarrow$ ] Here, the proof is done by a simple induction on $\lg l_{1}$. The main difficulty is that we have to recalculate all the indices in the functions for the new elements of ilist (where one element has been removed) in order to use the induction hypothesis.

Equivalence with Contejean. In [6], Contejean treats the same problem as ours but on lists. She bases her solution on the classical one presented in [20] but adds a base relation. It is defined as follows, using @ for list concatenation:

\section{Definition 13 (permcont, viewed inductively)}

$$
\begin{aligned}
& \forall R l_{1} l_{2}, \text { permcont }_{R} l_{1} l_{2} \\
& \Leftrightarrow\left\{\begin{array}{c}
l_{1}=l_{2}=[] \\
\exists a b l l_{1}^{\prime} l_{2}^{\prime}, l_{1}=a:: l_{1}^{\prime} \wedge l_{2}=l @ b:: l_{2}^{\prime} \wedge R a b \wedge \text { permcont }_{R} l_{1}^{\prime}\left(l @ l_{2}^{\prime}\right)
\end{array}\right.
\end{aligned}
$$

Remark 6. This definition is close to the spirit of Definition 7 but not with builtin symmetry since always the first element of $l_{1}$ is removed. Note that access to $b$ in $l @ b:: l_{2}^{\prime}$ is not a native operation of lists while it is in the spirit of ilist.

In order to validate the definitions we proposed here, we are going to show that iperm is equivalent to permcont (and then, by Theorem 2, ipermb will also be equivalent). As permcont applies to lists, we will need the conversion functions ilist2list and list2ilist (see Sect. 1.1] right after Definition 3).

Theorem 3. $\forall l_{1} l_{2}$, permcont ${ }_{R} l_{1} l_{2} \Rightarrow$ iperm $_{R}$ (list2ilist $l_{1}$ ) (list2ilist $l_{2}$ )

Proof. We work by induction on permcont ${ }_{R} l_{1} l_{2}$, with trivial base case. The inductive case gives us $a$ and $b$ that we remove from $l_{1}$ and $l_{2}$ using Definition 7 ,

We also show the other direction (this time using ilist2list) :

Theorem 4. $\forall l_{1} l_{2}$, iperm ${ }_{R} l_{1} l_{2} \Rightarrow$ permcont $_{R}$ (ilist2list $l_{1}$ ) (ilist2list $l_{2}$ )

Proof. We work by induction on iperm ${ }_{R}{ }_{1} l_{2} l_{2}$. The main technical difficulty here is that we need to obtain elements of the form $a:: l$ and $l_{1}^{\prime} @ b:: l_{2}^{\prime}$. The first one is easy (directly given by Definition 8 - Definition 7 would not be enough here) but for the second one we will need to manipulate the "right part" and the "left part" of an ilist, which is not so trivial.

\subsection{Comparison of the Obtained Notions}

We have presented with details four different definitions of permutations on ilist. All of them have been shown extensionally (i. e., pointwise) equivalent. Still, they are conceptionally quite different.

The first two, i. e., Definitions 7 and 8 are declarative in the sense that they do not say what a permutation is, and they are direct in the sense that they only use the concepts of ilist. We consider Definition 7 to capture the intuition and to be conceptually the definition of our choice (in the sequel, we will always work 
with Definition 7, unless specified otherwise). Definition 8 shares the same intuition of recursively removing pairs of elements, but with built-in extra uniformity for the possibility of choosing a pair. As mentioned before, this is good for proving transitivity but lacks symmetry in its construction, which is why we prefer Definition 7 as the reference definition. The variant with skeletons, iperm_skel, only makes explicit the information contained in a derivation according to Definition 7. Having this otherwise hidden information is necessary if several base relations are considered, as it is done in Lemma 3. The skeletons represent bijective functions on the indices but this information is kept totally implicit. Indeed, we only register indices corresponding to intermediate situations - not to the initial ilist.

Definition ipermb is based on an explicit representation of bijective functions on the indices. Surjectivity is quite delicate from a constructive point of view. And instead of requiring the existence of an inverse, it is better to exhibit it directly and make it part of the definition of bijectivity as done in bij. For ipermb to hold, one requires the existence of a bijection such that the elements pointed to by the pairs of indices according to the bijection are in the base relation. Conceptually, this separates the process of finding a permutation into first giving all the pairs of indices and second verifying that the elements corresponding to each pair indeed are in the base relation.

Finally, definition permcont given by Contejean immediately allows us to define a fifth notion of permutation:

Definition 14. $\forall l_{1} l_{2}$, ipermcont $_{R} l_{2} l_{2} \Leftrightarrow$ permcont $_{R}$ (ilist2list $\left.l_{1}\right)$ (ilist2list $\left.l_{2}\right)$

Since ilist2list is a bijection, ipermcont is extensionally equivalent to iperm, by Theorem 3 and Theorem 4 . However, as we have already explained, using lists in the coinductive definition of Graph is not an option. Therefore, we prefer a direct definition of permutations on ilist rather than through conversion between lists and ilist. Moreover, the basic notions on lists are the notions of head and tail, while getting the $n^{t h}$ element is not built into the structure. On the contrary, on ilist, we can get the $n^{\text {th }}$ element directly but it is not trivial to get the tail.

\section{A Relation on Graph Including Permutations on ilist}

In this section, we use iperm to define a relation on Graph. This relation will only solve the problem of permutation between children (see Fig. 1). A refinement that also solves the other problem (change in the point of view for the observation of the graph, see Fig. 2) is presented in Sect. 5.

We define the relation transformer GPerm using the same model as for Geq:

\section{Definition 15 (GPerm, Viewed Coinductively)}

$g_{1} g_{2}:$ Graph $T \quad R\left(\right.$ label $\left.g_{1}\right)\left(\right.$ label $\left.g_{2}\right) \quad$ iperm $_{\text {GPerm }_{R}}\left(\right.$ sons $\left.g_{1}\right)\left(\right.$ sons $\left.g_{2}\right)$ 


\subsection{An Unsuccessful Try with Guardedness as Implemented in Coq}

Proving that GPerm preserves reflexivity should be an easy exercise. Notice that this cannot be done by induction on $g$ in Graph $T$ since that type is coinductive. Since the proposition to be shown is coinductively defined, the proof naturally has to be carried out by coinduction.

Lemma 5 (GPerm Preserves Reflexivity). $\forall R$, Rreflexive $\Rightarrow \forall g$, GPerm $_{R} g g$

Proof (Coinductive proof). After assuming $R$ and its reflexivity, we try to show our goal by the cofix tactic of Coq. The coinductive hypothesis $H c$ is identical with our goal $\forall g, G P e r m_{R} g g$, and it suffices to prove for given $g$ :

$$
R \text { (label } g)(\text { label } g) \wedge \text { iperm }_{\text {GPerm }_{R}} \text { (sons g) (sons g) }
$$

- $R$ (label $g$ ) (label $g$ ) holds by reflexivity of $R$

- To prove iperm $_{\text {GPerm }_{R}}$ (sons $g$ ) (sons $g$ ) we may want to use the lemma that iperm preserves reflexivity. Thus, to prove iperm $_{\text {GPerm }_{R}}$ (sons g) (sons g), we would only have to prove that $\forall g, G P e r m_{R} g g$, which is $H c$. However, this proof is not correct for Coq because the use of $H c$ is not guarded (it should be used directly under a coinductive constructor and not through a lemma). So, we rather inline the proof of reflexivity of iperm $_{G P e r m_{R}}$ for the argument sons $g$. However, this part of the proof is by induction inside the initial coinduction (the corecursive call is in a recursive construction). Thus, the resulting proof does not pass the guardedness check of Coq.

ABORT PROOF.

This failure should not be surprising since it just repeats on the proof level the problems we would have had on the level of programming with Graph, had we not replaced the use of lists by ilist in its definition. In general, Coq support for coinductive types is quite comfortable, which is why the burden of working with ilist is compensated for by flexible coinductive definitions of graph structures. On the level of coinductive proofs about coinductively defined relations such as GPerm, this support does not seem of equal importance to us. Thus, we do not try to solve the issue of the rigid guardedness criterion of Coq as we did for Graph, but give up the use of the CoInductive command of Coq for getting a coinductive definition of GPerm. We rather define GPerm "manually" by an impredicative encoding, as shown next.

\subsection{Alternative Reading of the Coinductive Definition of GPerm}

We still view Definition [15] coinductively, but not according to the CoInductive keyword of Coq, as we did for all other coinductive definitions. We rather do this by an impredicative definition. This is based on Tarski's fixed point theorem that constructs a greatest fixed point in a complete lattice as the supremum of all of its post-fixed points. In the categorical picture, post-fixed points correspond to coalgebras, but in a complete lattice, we can even enforce finality without continuity. The Tarski construction can be replayed in polymorphic lambdacalculus as an impredicative encoding of greatest fixed points. All this is well 
explained in 22], together with historical remarks that, for least fixed points, the corresponding encodings go back to Böhm/Berarducci and Leivant.

While the CIC was an extension of polymorphic $\lambda$-calculus (hence, with impredicative universe Set) this impredicativity is now optional (so, we did not use it for Graph), but the universe of propositions Prop remains impredicative. In particular, we can form propositions by quantifying over all propositions or all relations, as they are building blocks of propositions. We use the wellknown impredicative construction on Prop instead of Set. We thus implement GPerm $_{R} g_{1} g_{2}$ by the proposition (with $\mathcal{R}$ ranging over relations on Graph $T$ ):

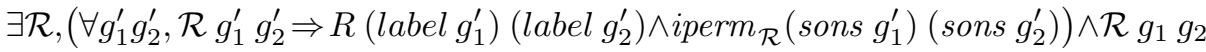

Lemma 6 (Coinduction Principle for GPerm). Let us assume

$\forall g_{1} g_{2}, \mathcal{R} g_{1} g_{2} \Rightarrow R$ (label $\left.g_{1}\right)$ (label $\left.g_{2}\right) \wedge$ iperm $_{\mathcal{R}}$ (sons $\left.g_{1}\right)$ (sons $g_{2}$ ) with $\mathcal{R}$ a relation on Graph $T$. Then, $\forall g_{1} g_{2}$, $\mathcal{R} g_{1} g_{2} \Rightarrow$ GPerm $_{R} g_{1} g_{2}$.

Proof. Trivial by implementation of GPerm.

Lemma 7 (Unfolding Principle for GPerm).

$\forall g_{1} g_{2}$, GPerm $_{R} g_{1} g_{2} \Rightarrow R\left(\right.$ label $\left.g_{1}\right)\left(\right.$ label $\left.g_{2}\right) \wedge$ iperm $_{\text {GPerm }}$ (sons $\left.g_{1}\right)\left(\right.$ sons $\left.g_{2}\right)$

Lemma 8 (Constructor for GPerm).

$\forall g_{1} g_{2}, R\left(\right.$ label $\left.g_{1}\right)\left(\right.$ label $\left.g_{2}\right) \wedge$ iperm $_{\text {GPerm }_{R}}$ (sons $\left.g_{1}\right)\left(\right.$ sons $\left.g_{2}\right) \Rightarrow$ GPerm $_{R} g_{1} g_{2}$

This final result corresponds to the way coinductive definitions are perceived for definitions by CoInductive in Coq. In our implementation, the coinduction principle is the obvious result, and the constructor the non-obvious (but wellknown for a long time by now). Therefore, these proofs are not shown here.

Remark 7. GPerm can also be captured in a Mendler-style coinduction (see [13]) that is directly expressible by CoInductive in Coq and also essentially uses impredicativity of Prop. This alternative gives a provably equivalent solution, as shown in our Coq development [17.

\subsection{Proof That GPerm Preserves Equivalence}

With the impredicative implementation of GPerm, Lemma 5 is now easy to prove. Proof. Assume that $R$ is reflexive. Instead of our goal $\forall g, G_{P e r m} g g$, we show the more general $\forall g_{1} g_{2}, g_{1}=g_{2} \Rightarrow$ GPerm $_{R} g_{1} g_{2}$, which is of the right format for Lemma 6, taking $\mathcal{R}:={ }_{\text {Graph }} T$. It suffices to show that $\mathcal{R} g_{1} g_{2}$ implies $R\left(\right.$ label $\left.g_{1}\right)\left(\right.$ label $\left.g_{2}\right) \wedge$ iperm $_{\mathcal{R}}$ (sons $\left.g_{1}\right)$ (sons $g_{2}$ ). Since $\mathcal{R}$ is Leibniz-equality, we may replace $g_{1}$ by $g_{2}$ everywhere. The first conjunct is then done by reflexivity of $R$, and the second follows from preservation of reflexivity by iperm.

GPerm also preserves symmetry and transitivity. For the former, the use of Lemma 6] is with $\mathcal{R}$ the "flipped" version of GPerm $_{R}$, hence with interchanged arguments, and uses the good flipping behaviour of iperm and Lemma 7.

For transitivity preservation, we set $\mathcal{R} g_{1} g_{2} \Leftrightarrow \exists g^{\prime}$, GPerm $_{R} g_{1} g^{\prime} \wedge$ GPerm $_{R} g^{\prime} g_{2}$ and use Lemma 6] for it. Besides Lemma 7, this requires a quite special form of transitivity of iperm whose proof must be based on Definition 8 and Theorem 1 . iperm $_{R_{1}} l_{1} l^{\prime} \wedge$ iperm $_{R_{2}} l^{\prime} l_{2} \Rightarrow$ iperm $_{R_{3}} l_{1} l_{2}$ with $R_{3} t_{1} t_{2} \Leftrightarrow \exists t^{\prime}, R_{1} t_{1} t^{\prime} \wedge R_{2} t^{\prime} t_{2}$ 


\section{An Equivalent Approach Based on Observations}

As we saw in Sect. 3.1, the problem was the embedding of inductive proofs (the existence of a permutation) in a coinductive proof that GPerm holds. This could be overcome by the impredicative encoding of GPerm, but there is also the well-known option of abandoning coinduction in favour of some observational equivalence. Here, the idea is to use inductive trees (the finite counterpart to Graph) to represent the unfolding of elements of Graph until a certain depth. Then, we define a relation transformer on these trees using iperm, and we prove that it preserves equivalence. To "observe" until a depth $n$ means cutting off the graphs all the structure beyond depth $n$, which yields such inductive trees, so we can relate them by the relation on trees (obviously, we expect the relation to be decidable). If the relation holds for all depths, the graphs are considered "observationally equal". Thus, on the logical side, we only work on inductive structures (while the graphs are still coinductive structures).

Finally, adding a version of the infinite pigeonhole principle as an axiom to Coq (that could in turn be justified by the classical law of excluded middle), we prove that observational equivalence is equivalent to GPerm (for any base relation). Therefore, we get an alternative proof that GPerm preserves equivalence.

\subsection{Definitions Based on Inductive Trees}

We define the type of inductive trees (we call it iTree) as follows:

\section{Definition 16 (iTree, Viewed Inductively)}

$$
\frac{t: T \quad l: i l i s t(i \text { Tree } T)}{m k_{\text {_iTree }} t l: i \text { Tree } T}
$$

We call labT and sonsT the two projection functions on iTree. We use $t_{1}$ and $t_{2}$ to denote elements of iTree $T$.

Now, we can define a relation on iTree using the permutation relation on ilist. We define it in analogy with GPerm, but inductively:

\section{Definition 17 (TPerm, Viewed Inductively)}

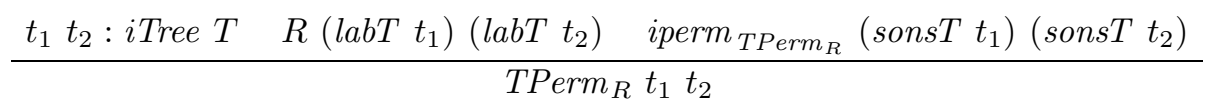

To prove that TPerm preserves equivalence, we reason by induction. As iperm is also inductive, the proofs are straightforward and do not present any difficulties.

As we have said, the purpose of using inductive trees built with the same pattern as Graph is to capture the unfolding of an element of Graph until a depth $n$. Therefore, we have to define a function that transforms an element of Graph into an element of iTree. We call this function G2iT.

\section{Definition 18 (G2iT, Defined Recursively)}

$$
\begin{aligned}
& \text { G2iT }: \forall T, \mathbb{N} \rightarrow \text { Graph } T \rightarrow \text { iTree } T \\
& \text { G2iT T } 0 \mathrm{~g}:=\text { mk_iTree }(\text { label } g) \llbracket \rrbracket \\
& \text { G2iT } T(n+1)\left(m k_{-} \text {Graph } t l\right):=m k_{-} \text {iTree } t(\text { imap }(\text { G2iT } T n) l)
\end{aligned}
$$


where 【』 stands for the empty ilist and imap is the ilist analogue of the map function on lists (see [16]) and has type $\forall T U,(T \rightarrow U) \rightarrow$ ilist $T \rightarrow$ ilist $U$.

We also define a notation to say that two elements of Graph extracted up to level $n$ are equivalent through TPerm. We call it $\equiv$ :

Definition $19 \forall n g_{1} g_{2}, g_{1} \equiv_{R, n} g_{2} \Leftrightarrow \operatorname{TPerm}_{R}\left(\right.$ GRiT $\left.n g_{1}\right)\left(\right.$ GRiT $\left.n g_{2}\right)$

Lemma 9 (Recursive description of $\equiv_{R, n}$ as a function of $n$ )

$$
\forall g_{1} g_{2}, g_{1} \equiv_{R, 0} g_{2} \Leftrightarrow R \text { (label } g_{1} \text { ) (label } g_{2} \text { ) }
$$

$\forall g_{1} g_{2}, g_{1} \equiv_{R, n+1} g_{2} \Leftrightarrow R\left(\right.$ label $\left.g_{1}\right)\left(\right.$ label $\left.g_{2}\right) \wedge$ iperm $\equiv_{R, n}\left(\right.$ sons $\left.g_{1}\right)\left(\right.$ sons $\left.g_{2}\right)$

Remark 8. As should be expected from a relation based on finite observations, $\equiv_{R, n}$ preserves decidability (of $R$ ). The proof is by induction on $n$ using Lemmas 1 and 9 .

Our goal here is to prove that, if for all $n$, two elements of Graph transformed to iTree by G2iT $n$ are equivalent through TPerm, then they are equivalent through GPerm and vice versa. We first define the relation on Graph that uses TPerm. We want to prove that it is equivalent to GPerm. We call it GTPerm.

Definition 20 (GTPerm). $\forall g_{1} g_{2},\left(\right.$ GTPerm $\left._{R} g_{1} g_{2} \Leftrightarrow \forall n, g_{1} \equiv_{R, n} g_{2}\right)$

One shows that GTPerm preserves equivalence using the fact that TPerm does.

\subsection{Main Theorem}

Theorem 5. $\forall g_{1} g_{2}$, GPerm $_{R} g_{1} g_{2} \Leftrightarrow$ GTPerm $_{R} g_{1} g_{2}$

The proof extends until the end of this section and makes use of Axiom 1 below. Proof

[Direction $\Rightarrow]$ This is proved by induction on $n$ (depth of observation of $g_{1}$ and $\left.g_{2}\right)$ using Lemma 9 .

[Direction $\Leftarrow$ ] This direction is much harder, and needed concepts and results will be introduced after showing the overall structure. We do it as a coinductive proof, i. e., we apply Lemma 6. Obviously, we use it with $\mathcal{R}:=\operatorname{GTPerm}_{R}$. Given $g_{1}, g_{2}$ and GTPerm $R g_{1} g_{2}$, we have to show $R$ (label $\left.g_{1}\right)$ (label $\left.g_{2}\right)$ and iperm $_{\text {GTPerm }}$ (sons $g_{1}$ ) (sons $g_{2}$ ). For the first part, we use the definition of GTPerm $_{R}$ with $n:=0$ and the base case of Lemma 9. The second part is guaranteed by Lemma 10 .

Lemma 10. $\forall g_{1} g_{2}$, GTPerm $_{R} g_{1} g_{2} \Rightarrow$ iperm $_{\text {GTPerm }_{R}}\left(\begin{array}{lll}\text { sons } g_{1}\end{array}\right)\left(\begin{array}{lll}\text { sons } g_{2}\end{array}\right)$

[Proof of Lemma 10] Actually, here, the main challenge is a problem of continuity. Indeed, unfolding the definition of GTPerm, we obtain the goal:

$$
\forall g_{1} g_{2},\left(\forall n, g_{1} \equiv_{R, n} g_{2}\right) \Rightarrow \text { iperm }_{\cap_{n} \equiv_{R, n}} \text { (sons } g_{1} \text { ) (sons } g_{2} \text { ) }
$$


So this actually means that we can "fix" a permutation for (sons $g_{1}$ ) and (sons $g_{2}$ ) that will be valid for any depth of extraction through G2iT. This is not trivial, because some permutations may be valid until a certain depth but not afterwards. For example, in Fig. 3, only the permutation symbolized by the thick plain arrows would be valid for the level with depth $=2$. The thick dashed one would be valid for depth $=1$ but not afterwards (given standard equality on the labels). However, in the opposite direction, there is no problem. Indeed, if a permutation is valid at a certain depth $n$, then for all shallower depths $m(m \leq n)$, this same permutation is valid. We express this result only partly in Lemma 11] since we do not capture in what sense this is the "same permutation". The proof is not detailed here.

Lemma 11. $\forall g_{1} g_{2} n m, m \leq n \wedge g_{1} \equiv_{R, n} g_{2} \Rightarrow g_{1} \equiv_{R, m} g_{2}$

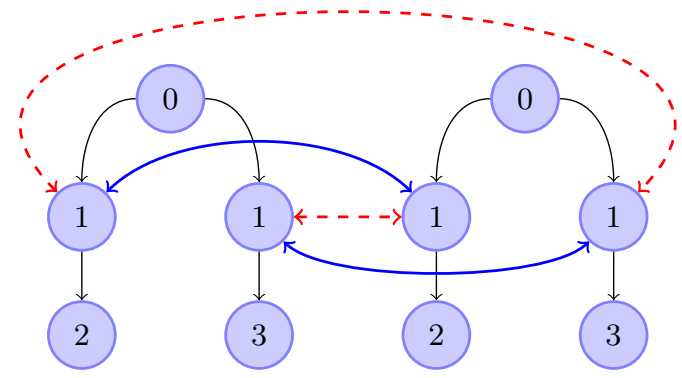

Fig. 3. Graphs with various possibilities of permutations on the first level

To prove Lemma 10, we use a specific formulation of the infinite pigeonhole principle. It informally states that if an infinity of items is put into a finite number of holes, then at least one hole must contain an infinity of items. Although obviously true, this principle has no constructive justification since no finite observation of the infinite process of putting the items into the holes allows to determine which hole is used infinitely often.

For our problem, the infinitely many items are the unfolding levels (for the elements of Graph) and the holes are the possible permutations (as sons $g_{1}$ and sons $g_{2}$ are finite, the number of possible permutations also is, as we only consider the permutations on the top level).

However, this requires us to be able to manipulate permutations. Hence, we have to use the definition of iperm_skel given in Sect.2.2. We can do this directly since we showed that it was equivalent to iperm.

The infinite pigeonhole principle will only be needed for the finite types of the form skel_type $m$. We have to assume it as an axiom on top of the intuitionistic type theory CIC that underlies Coq. It could be justified by the classical law of excluded middle which is consistent with the CIC.

\section{Axiom 1 (Infinite Pigeonhole Principle).}

$$
\begin{aligned}
\forall m \forall P: \mathbb{N} \rightarrow \text { skel_type } m & \rightarrow \text { Prop, }(\forall n \exists s: \text { skel_type } m, P n s) \rightarrow \\
& \exists s_{0}: \text { skel_type } m,\left(\forall n \exists n^{\prime}, n^{\prime} \geq n \wedge P n^{\prime} s_{0}\right)
\end{aligned}
$$


Here, we use the letter $P$ for the relation that describes which "pigeon" $n$ "goes" into which "hole"/skeleton $s$. We can now come back to Lemma 10 itself. We want to prove that:

$$
\text { GTPerm }_{R} g_{1} g_{2} \Rightarrow \text { iperm }_{\text {GTPerm }} \text { (sons } g_{1} \text { ) (sons } g_{2} \text { ) }
$$

It is easy to prove $l g\left(\right.$ sons $\left.g_{1}\right)=\lg \left(\right.$ sons $\left.g_{2}\right)$ (call its proof $\left.H_{l g}\right)$ using the definition of GTPerm with $n$ :=1. We can also prove the following assertion $H_{1}$ :

$$
\left.\left.\forall n \exists s \text { : skel_type }\left(\text { lg (sons } g_{1}\right)\right) \text {, iperm_skel } \equiv_{R, n} \text { (sons } g_{1} \text { ) (sons } g_{2}\right) H_{l g} s
$$

Indeed, for argument $n$, the definition of TTPerm $_{R} g_{1} g_{2}$ can be used at depth $n+1$, and Lemma9 allows to infer iperm $_{\equiv_{R, n}}$ (sons $g_{1}$ ) ( sons $_{2}$ ), from which we get the skeleton by Lemma 2 .

The application of the pigeonhole principle (Axiom 1) gives us the "good" permutation skeleton $s_{0}$ for infinitely many levels, as expressed in the property

$$
\left.\forall n \exists n^{\prime}, n^{\prime} \geq n \wedge \text { iperm_skel } \equiv_{R, n^{\prime}} \text { (sons } g_{1} \text { ) (sons } g_{2}\right) H_{l g} s_{0} \quad\left(H_{2}\right)
$$

In fact, $s_{0}$ is already the "good" skeleton for all levels: using Lemma 2 we can change the goal of the proof to:

$$
\text { iperm_skel } \left.\text { GTPerm }_{R} \text { (sons } g_{1} \text { ) (sons } g_{2}\right) H_{l g} s_{0}
$$

and by Lemma 3, we can transform it again into

$$
\left.\forall n, \text { iperm_skel } \equiv_{R, n} \text { (sons } g_{1} \text { ) (sons } g_{2}\right) H_{l g} s_{0}
$$

Given $n$, we choose $n^{\prime}$ according to $H_{2}$. Then, by Lemma 11] $\equiv_{R, n^{\prime}}$ is a subrelation of $\equiv_{R, n}$. Therefore, using the monotonicity of iperm_skel in its relation argument, we obtain the goal from the main property of $n^{\prime}$ that $H_{2}$ guarantees.

This ends the proof of Lemma 10 and hence completes that of Theorem 5.

\section{The Final Relation}

The preceding two sections solve the issue of permutations in the children. We are going to integrate it in a solution that also solves the issue of change of root.

\subsection{The Idea}

Graph allows to represent single-rooted connected graphs, i. e., there is a path to any node of the graph from the root. If we are able to change the "point of view", i. e., to represent the same graph but with another node as the root (see Fig. 2), then there is a path from the new root to the old one and inversely.

Remark 9. This change is only allowed for a "general" view, not for the inner nodes. For example, we do not want the graphs of Fig. 4 to be equivalent.

We will call $g_{1}$ and $g_{2}$ the two elements of Graph that represent the same graph but with a different root. Saying that there is a path from $g_{1}$ to $g_{2}$ and from $g_{2}$ to $g_{1}$ means that $g_{1}$ is "included" in $g_{2}$ and that $g_{2}$ is "included" in $g_{1}$. In [16] 

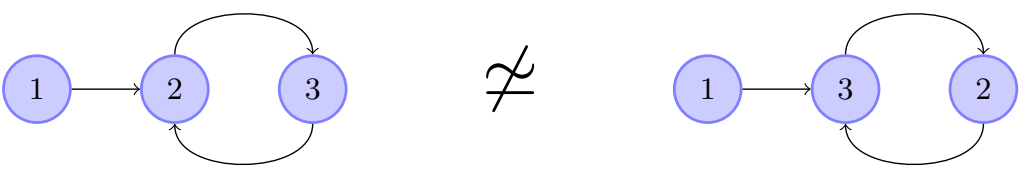

Fig. 4. Graphs with inner cycle turned

we have presented a notion of strict inclusion that we have called GinG. This inclusion was built using Geq. Here, we want to add permutations, so we want to have the same kind of relation but using GPerm. So we can define it in a general way (for any relation on Graph) and then we will instantiate it for GPerm. We define it as follows (with $R_{G}$ a relation on Graph $T$ ):

\section{Definition 21 ( $\operatorname{Gin} G^{*}$, Viewed Inductively)}

$$
\frac{R_{G} g_{\text {in }} g_{\text {out }}}{{\operatorname{Gin} G_{R_{G}}^{*} g_{\text {in }} g_{\text {out }}}_{\operatorname{Gin} G_{R_{G}}^{*} g_{\text {in }}\left(f c t\left(\text { sons } g_{\text {out }}\right) i\right)}}
$$

To find properties of $\operatorname{Gin} G^{*}$, we need to know more about the behaviour of $R_{G}$ regarding propagation towards the children. We instantiate Gin $^{*}$ for GPerm:

Definition 22 (GinGP). Gin $G P_{R}:=\operatorname{Gin}_{G P e r m_{R}}^{*}$

For GinGP, we can prove various properties among which preservation of transitivity and compatibility with relation GPerm $_{R}$ in its two arguments.

\subsection{GeqPerm}

Using this relation of inclusion, we can now define the final relation on Graph that takes into account permutations over the children and a change in the point of view of the representation. We call it GeqPerm. It checks that the first element of Graph is included in the second, and inversely. The case when there is no change in the point of view for the observation is managed by the fact that Gin $^{*}$ preserves reflexivity (first case of the definition). We define GeqPerm as follows:

\section{Definition 23 (GeqPerm)}

$$
\forall g_{1} g_{2}, \text { GeqPerm }_{R} g_{1} g_{2} \Leftrightarrow \operatorname{GinGP}_{R} g_{1} g_{2} \wedge \operatorname{GinGP}_{R} g_{2} g_{1}
$$

As Gin GP preserves reflexivity and transitivity, GeqPerm preserves equivalence. It is now possible to show that the graphs of Fig. 1 and Fig. 2] are equivalent and that the graphs of Fig. 4 are not. The proofs that two elements of Graph are not equivalent may be tedious because we have to test all possible permutations (in the example of Fig. 4 the nodes only have one child, so the proof is short). Of course, one can also mix the features of Fig. 1 and Fig. 2.

Remark 10. We now have at our disposal the relation on Graph that we would natively have obtained with a standard set-theoretic definition of graphs. But, we have the computational advantages of our constructive definitions. 


\section{Conclusions}

In this paper, we have presented and compared various solutions to capture permutations on ilist modulo some possibly undecidable base relation $R$ on the elements of the ilist. This was crucial since decidability cannot be guaranteed (not even expected) for our graph representation Graph. We have shown that these relation transformers preserve equivalence (map equivalence relations to equivalence relations). And we proved that they are all equivalent. Then, we have integrated one of these relations (the one that we considered to capture best the intuition of permutations) into a relation GPerm to include permutations over children. Using ilist instead of lists was needed since with lists, the coinductive representation of graphs would not have allowed programming in Coq.

We have also presented a solution that allows us to observe our graphs on finite structures (the inductive trees $i$ Tree) via the function G2iT that converts elements of Graph to elements of iTree up to a certain depth. We provide a relation transformer TPerm for iTree in analogy with what we did for Graph, including permutations over the children of the elements of $i$ Tree. We can thus formulate that two graphs are "bisimilar" up to any depth by reference to G2iT and TPerm, which yields the relation GTPerm. For a decidable base relation, we obtained that "bisimilarity" up to any given depth is decidable. We have included into the main text a proof of equivalence of GPerm with GTPerm (Theorem 5), hence between the coinductive concept and the one based on observations.

Finally, we included our permutation-aware relation on Graph into a wider relation taking into account changes in the point of view of the representation of the elements of Graph (GeqPerm). We proved that this final relation (transformer) preserves equivalence and that it indeed solved the issues presented in Sect. 1.2.

In the course of our research we observed that even for lists, the standard library would not have solved our problems concerning permutations parameterized freely by a possibly undecidable relation (see [20] and 21]). We are currently working on an extension of Contejean's $[6$ in the style of our solutions for ilist.

If we chose to ignore multiplicities in ilist, we could define a new relation corresponding to the equivalence between two sets. This could be an interesting direction to investigate to obtain an even more liberal relation on graphs.

Berger [3] uses an embedded inductive definition in a coinductive one for the purpose of real number computability, with extraction of Haskell programs. We wonder if his work could be formalized in Coq using the methods presented here.

Finally, it would be interesting to extend GeqPerm to a wider class of graphs covering also non-connected graphs, e. g., by using the notion of forest of graphs.

Acknowledgements. We warmly thank our reviewers for their attentive reading and interesting propositions and challenges.

\section{References}

1. Altenkirch, T.: A Formalization of the Strong Normalization Proof for System F in LEGO. In: Bezem, M., Groote, J.F. (eds.) TLCA 1993. LNCS, vol. 664, pp. 13-28. Springer, Heidelberg (1993) 
2. Berardi, S., Damiani, F., de'Liguoro, U. (eds.): TYPES 2008. LNCS, vol. 5497. Springer, Heidelberg (2009)

3. Berger, U.: From coinductive proofs to exact real arithmetic: theory and applications. Logical Methods in Computer Science 7(1) (2011)

4. Bertot, Y., Komendantskaya, E.: Using structural recursion for corecursion. In: Berardi et al. [2], pp. 220-236

5. Chlipala, A.: In Coq club thread "is Coq being too conservative?" (January 2010), https://sympa-roc.inria.fr/wws/arc/coq-club/2010-01/msg00089.html

6. Contejean, E.: Modeling Permutations in CoQ for Coccinelle. In: Comon-Lundh, H., Kirchner, C., Kirchner, H. (eds.) Jouannaud Festschrift. LNCS, vol. 4600, pp. 259-269. Springer, Heidelberg (2007)

7. Coquand, T.: Infinite Objects in Type Theory. In: Barendregt, H., Nipkow, T. (eds.) TYPES 1993. LNCS, vol. 806, pp. 62-78. Springer, Heidelberg (1994)

8. Dams, C.: In Coq club thread "is Coq being too conservative?" (January 2010), https://sympa-roc.inria.fr/wws/arc/coq-club/2010-01/msg00085.html

9. Danielsson, N.A.: Beating the productivity checker using embedded languages. In: Bove, A., Komendantskaya, E., Niqui, M. (eds.) Proceedings Workshop on Partiality and Recursion in Interactive Theorem Provers. EPTCS, vol. 43, pp. 29-48 (2010)

10. Danielsson, N.A., Altenkirch, T.: Subtyping, Declaratively. In: Bolduc, C., Desharnais, J., Ktari, B. (eds.) MPC 2010. LNCS, vol. 6120, pp. 100-118. Springer, Heidelberg (2010)

11. Giménez, E., Castéran, P.: A tutorial on [co-]inductive types in Coq (2007), http://www.labri.fr/perso/casteran/RecTutorial.pdf

12. McBride, C., McKinna, J.: The view from the left. Journal of Functional Programming 14(1), 69-111 (2004)

13. Nakata, K., Uustalu, T.: Resumptions, weak bisimilarity and big-step semantics for while with interactive I/O: An exercise in mixed induction-coinduction. In: Aceto, L., Sobocinski, P. (eds.) SOS. EPTCS, vol. 32, pp. 57-75 (2010)

14. Nakata, K., Uustalu, T., Bezem, M.: A Proof Pearl with the Fan Theorem and Bar Induction - Walking through Infinite Trees with Mixed Induction and Coinduction. In: Yang, H. (ed.) APLAS 2011. LNCS, vol. 7078, pp. 353-368. Springer, Heidelberg (2011)

15. Niqui, M.: Coalgebraic reasoning in Coq: Bisimulation and the lambda-coiteration scheme. In: Berardi et al. [2], pp. 272-288

16. Picard, C., Matthes, R.: Coinductive graph representation: the problem of embedded lists. Electronic Communications of the EASST 39, 24 pages (2011)

17. Picard, C., Matthes, R.: Formalization in Coq for this article (2012), http://www.irit.fr/ Celia.Picard/Coq/Permutations/

18. Prince, R., Ghani, N., McBride, C.: Proving Properties about Lists Using Containers. In: Garrigue, J., Hermenegildo, M.V. (eds.) FLOPS 2008. LNCS, vol. 4989, pp. 97-112. Springer, Heidelberg (2008)

19. The Coq Development Team: The Coq Proof Assistant Reference Manual, http://coq.inria.fr

20. The Coq Development Team: The Coq Proof Assistant Standard Library, http://coq.inria.fr/stdlib/Coq.Sorting.Permutation.html

21. The Coq Development Team: The Coq Proof Assistant Standard Library, http://coq.inria.fr/stdlib/Coq.Sorting.PermutSetoid.html\#permutation

22. Uustalu, T., Vene, V.: Least and greatest fixed points in intuitionistic natural deduction. Theoretical Computer Science 272, 315-339 (2002) 